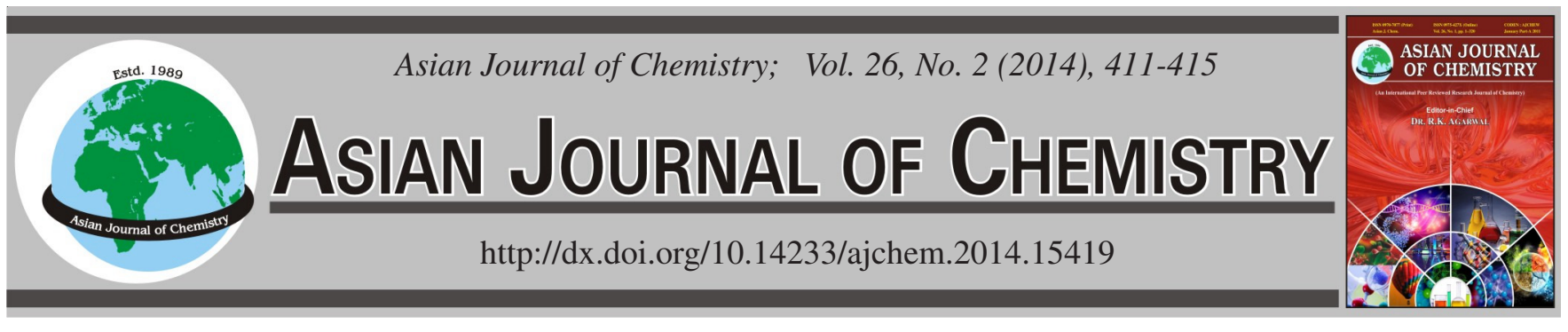

\title{
Effect of Two Different Sulfamethazine Addition Methods on Psychrophilic Anaerobic Digestion of Swine Wastewater
}

\author{
Lu Huang ${ }^{1}$, Ruipeng Guo ${ }^{1}$, JuAnboo Liang $^{3}$, Baohua Ma $^{4}$, Xindi Liao $^{1,2}$ and Yinbao Wu ${ }^{1,2, *}$
}

${ }^{1}$ College of Animal Science, South China Agricultural University, Guangzhou 510642, P.R. China

${ }^{2}$ Ministry of Agriculture Key Laboratory of Tropical Agricultural Environment, South China Agricultural University, Guangzhou 510642, P.R. China

${ }^{3}$ Institute of Tropical Agriculture, Universiti Putra Malaysia, Serdang 43400, Malaysia

${ }^{4}$ Nanhai Entry-Exit Inspection and Quarantine Bureau, Foshan 528200, P.R. China

*Corresponding author: E-mail: wuyinbao@scau.edu.cn

Received: 4 March 2013;

Accepted: 11 June 2013;

Published online: 15 January 2014;

AJC-14555

\begin{abstract}
Veterinary antibiotic residue in manure is one of the most important factors which have an impact on the anaerobic digestion. This article studied the effect of sulfamethazine (SM-2) on psychrophilic anaerobic digestion of swine manure by two adding methods, addition of manure from swine fed with SM-2 in diet and direct addition of SM-2 with blank manure, under two adding concentrations of SM-2 (2.38 and $1.24 \mathrm{mg} / \mathrm{g}$ dry swine manure). Anaerobic digestion experiment was conducted at $20{ }^{\circ} \mathrm{C}$ in laboratory digesters with the effective working volume of $1.3 \mathrm{~L}$. Methane production of each digester was measured by displacement method daily. Grad samples were collected to determine the concentration of SM-2, pH, chemical oxygen demand (COD) in slurry and organic matter in biogas residue. The results showed that the concentration of SM-2 in anaerobic system fed with manure containing SM-2 was lower than that fed with blank manure and SM-2 under the same addition of SM-2 and the degradation rate of SM-2 in the high concentration treatment was faster than that in the low concentration treatment. Compared with the control group, the treatments added with SM-2 had no significant difference in methane production, COD in slurry and organic matter in biogas residue, but adding SM-2 and blank manure improved pH of swine slurry in SM-2 adding period. Due to the different degradation rate of SM-2 in anaerobic digestion between the two adding methods of SM-2, using manure collect from animals administered with SM-2 is more appropriate to assess the effect of SM-2 on anaerobic digestion.
\end{abstract}

Keywords: Sulfamethazine, Swine manure, Anaerobic digestion.

\section{INTRODUCTION}

Sulfamethazine (SM-2) is a broad-spectrum antibiotic of the family of the sulfonamides, which inhibits protein synthesis in many common gram-positive and gram-negative bacteria, chlamydia, rickettsie, etc. It is widely administrated to farm animals to control intestinal and respiratory infections. Previous studies showed there were high sulfonamides residues in swine manure and wastewater. Mellon et al. ${ }^{1}$ reported that swine manure contained $133 \mathrm{mg} / \mathrm{kg}$ mixture of chlortetracycline (CTC), sulfonamides and penicillin. The content of mixture including chlortetracycline, sulfamethazine and penicillin was $140 \mathrm{mg} / \mathrm{L}$ in swine wastewater. Zhao et al. ${ }^{2}$ reported that the SM-2 maximum residue in chicken manure was $6.04 \mu \mathrm{g} / \mathrm{g}$ and there was significant difference among regions. For the broadspectrum inhabitation to microorganism, the ecotoxicological effect of SM-2 was more and more concerned recently.

Anaerobic fermentation can degrade organic matter effectively and produce valuable biogas as clean energy. The process breaks up the water-insoluble organic matter into the water-soluble matter which provides an important prerequisite for reduction of offensive odor generated during waste storing and kills or inhibits a variety of parasites and pathogens. Because of the above benefits, anaerobic fermentation has been widely used in China for the treatment of pig waste. The Chinese Agriculture Ministry gives emphasis to construct biogas demonstration projects in large and medium livestock farm for waste treatment and targeted that biogas projects would reach $85 \%$ of the 8,000 livestock farms throughout China in 2010. The efficiency of anaerobic digestion was affected by various factors including veterinary antibiotics residue in the waste. When veterinary antibiotics entered anaerobic reactor with waste, they may cause inhibition or failure during the anaerobic digestion. Since the early 1980s, the inhibitory effect of veterinary antibiotics on anaerobic digestion in livestock waste has received much attentions ${ }^{3}$. Recently more researchers found that the inhibitory effects were related to the species and concentration of veterinary antibiotics. Therefore, focuses were 
given to measure the effect of different veterinary antibiotics under different adding concentration, through determination of veterinary drugs and some functional parameters in anaerobic digestion ${ }^{4-6}$.

Two research methods were used to study the effect of veterinary antibiotics on anaerobic digestion; one is direct addition of the antibiotics to anaerobic digestion systems and another is using manure collect from animal treated with antibiotics. Sankvist et al. ${ }^{7}$ suggested the latter method is more appropriate. However, both methods were used in recent researches to study the effect of veterinary antibiotics on anaerobic digestion. Till now, it is unknown if there are differences in the two mentioned methods and what the differences are. So this study examined the effect of SM-2 on psychrophilic anaerobic digestion in model semi-batch reactors using both methods under two concentrations of SM-2. The results were compared to examine the influence of research method on toxicology effect of SM-2 in anaerobic digestion.

\section{EXPERIMENTAL}

Swine manure collection: Manure samples were collected from a breeding pig farm in Zengcheng, Guangzhou, China. 78 growing swine of about $34 \mathrm{~kg}$ liveweight fed with antibiotic free diet for the last 28 days were used to supply the experimental manure. Swine were divided into 2 groups consisting of 3 replications of 13 pigs each replication. Swine in the control group were continued to receive antibiotic free diet while the other group was fed with the same diet which contained $0.2 \mathrm{~g} \mathrm{SM}-2 / \mathrm{d} / \mathrm{kg}$ body weight, as permitted by the Veterinary Pharmacopoeia of the People Republic of China ${ }^{8}$. About $2 \mathrm{~kg}$ pooled manure excreted by the swine from each group were collected daily and stored at $-20^{\circ} \mathrm{C}$ for subsequent used. The samples were later determined for SM-2 concentrations and the manure containing maximum and average concentration of SM-2 were selected for the anaerobic digestion.

Anaerobic digesters: Experiment was conducted in laboratory model anaerobic digesters (Fig. 1) ${ }^{9}$. Each digester was a three-neck glass reactor with a working volume of $1.3 \mathrm{~L}$. The three openings of the digester were sealed with rubber stoppers. Swine slurry sample was collected from the right opening, while the fresh slurry was added into the digester through the left opening. The volume of biogas was obtained by drainage method as follows: the biogas generated from the system flowed into a $500 \mathrm{~mL}$ absorbing flask filled with 0.5 $\mathrm{mol} / \mathrm{L} \mathrm{NaOH}$ solution and the volume of $\mathrm{NaOH}$ solution being replaced by the incoming methane was push out of the absorbing flask into receiver flask, the volume of biogas was obtained by measuring the volume of $\mathrm{NaOH}$ solution in receiver flask.

To prepare the digester for the experiment, each digester was filled with $100 \mathrm{~g}$ swine manure collected from swine fed with antibiotics free diet. Slurry $(100 \mathrm{~mL})$ from anaerobic wastewater

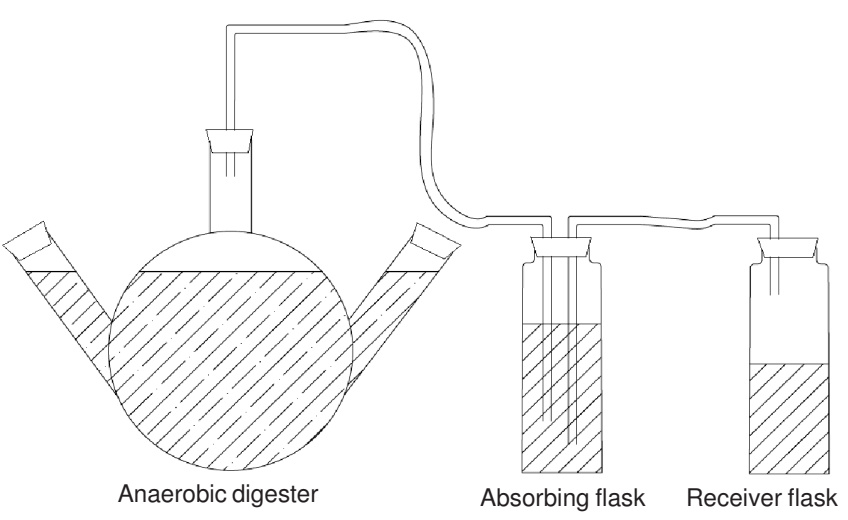

Fig. 1. Model anaerobic digesters

treatment pond from the same farm was added into the digester as inoculum, followed by $800 \mathrm{~mL}$ of water. After the digester was filled as described above, the headspace was flushed with nitrogen gas to maintain an anaerobic condition. Every day at 08:00 AM, $100 \mathrm{~mL}$ anaerobic slurry from each digester was poured out of the digester via the right opening and reloaded with an equal volume of antibiotic free manure with its moisture content adjusted to $90 \%$ via the left opening; the digesters were only used for the experiment when the production of methane was steady.

Experiment design: The experiment consisted of a control and four treatment groups. The treatment groups were designed as a combination of two research methods and two levels of SM-2 as shown in Table-1.

Each treatment was replicated using 3 digesters incubated in a biochemical incubator set at $20 \pm 0.5^{\circ} \mathrm{C}$. The trial lasted 28 days. In the control, $100 \mathrm{~mL}$ of antibiotic free manure slurry was added daily throughout the 28 days, while in the treatment groups, the manure containing SM-2 was added for the initial 7 days consecutively as the SM- 2 adding period, followed by antibiotic free manure for the next 21 days as the SM-2 withdrawing period.

Sample collection and analysis: Methane production of each digester was measured at 08:00 AM and 6:00 PM everyday. Swine slurry sample $(50 \mathrm{~mL})$ was collected from each digester on days $0,1,2,3,4,5,6,7,8,10,12,14,17,21$ and 28, used for determination of $\mathrm{pH}, \mathrm{COD}$ and $\mathrm{SM}-2.50 \mathrm{~mL}$ sample collected on days 0,8 and 28 were dried for determination organic matter.

Detection of SM-2 in swine slurry: Swine slurry sample $(5 \mathrm{~mL})$ was extracted twice with $5 \mathrm{~mL} 0.01 \mathrm{M} \mathrm{Na} \mathrm{NaDTA}_{2}$ by vortexing $30 \mathrm{~s}$, followed by concussion for $10 \mathrm{~min}$ at 3000 rpm. After each extraction, the extracts were subjected to centrifugation ( $12000 \mathrm{rpm}$ for $10 \mathrm{~min}$ at $5^{\circ} \mathrm{C}$ ). The supernatants were pooled in new centrifuge tube, centrifugated again and filtered through $2.5 \mathrm{~mm}$ filter paper by Buchner funnel and passed through prewashed Phenomenex Strata-X-CW $\mathrm{C}_{18}$

TABLE-1

EXPERIMENTAL TREATMENT GROUPS OF ANAEROBIC DIGESTION TRIAL

\begin{tabular}{ccc}
\hline Group & SM-2 concentration $(\mathrm{mg} / \mathrm{g}$ DM) in swine manure & Description \\
\hline 1 & 0 & Adding with antibiotic free manure \\
2 & 2.38 & Adding with manure from medicated swine \\
3 & 2.38 & Adding with SM-2 and antibiotic-free manure \\
4 & 1.24 & Same as group 2 \\
5 & 1.24 & Same as group 3 \\
\hline
\end{tabular}


cartridges. The cartridges were prewashed with $1 \mathrm{~mL}$ of methanol followed by $1 \mathrm{~mL}$ of distilled water. After the extracts were loaded, the cartridges were flushed with $1 \mathrm{~mL}$ distilled water, followed by sample elution using $1 \mathrm{~mL}$ methanol and $1 \mathrm{~mL}$ $2 \%$ formic acid + methanol solution. Eluents were filtration with $0.22 \mu \mathrm{m}$ filter prior to analysis by HPLC (Waters 600 ControllerWaters 717 plus Auto-sampler, Waters 600E-2487 Dual ë Absorbance Detector, USA) with a Kromasil $\mathrm{C}_{18}$ column $(250 \mathrm{~mm} \times 4.6 \mathrm{~mm}, 5 \mu \mathrm{m}, 100 \mathrm{~A})$ at $30{ }^{\circ} \mathrm{C}$. The detection wavelength was $275 \mathrm{~nm}$ and the injection volume was $10 \mu \mathrm{L}$. The mobile phase was acetonitrile-5\% acetic acid (10:90, v/v) and the flow rate was set at $1.0 \mathrm{~mL} / \mathrm{min}$. Under the above conditions, SM-2 could be detached from other components in swine slurry. The retaining time of SM-2 was $7.97 \mathrm{~min}$ with a detection limit of $0.1 \mu \mathrm{g} / \mathrm{mL}$ and recoveries of SM-2 were $75 \%$.

Statistical analysis: The statistical analyses were performed using SPSS 13.0. ANOVA and Turkey test were assumed to compare the variances of different groups. Significance was accepted at probabilities $(p)$ of 0.05 level for all analysis.

\section{RESULTS AND DISCUSSION}

Concentrations of SM-2 in swine slurry: Concentrations of SM-2 in swine slurry during the anaerobic digestion were shown in Fig. 2. After addition of SM-2, its concentration increased in the four experimental groups while no SM-2 was detected in the group 1 (control group). The average SM-2 concentration in group 2-5 was 269.49, 87.90, 130.93 and $78.79 \mathrm{mg} / \mathrm{L}$ in SM-2 adding period (the initial 7 days), respectively. The SM-2 concentration of experimental groups reduced gradually in SM-2 withdrawing period. SM-2 could not be detected after the 4th day of withdrawals in all treatment groups.

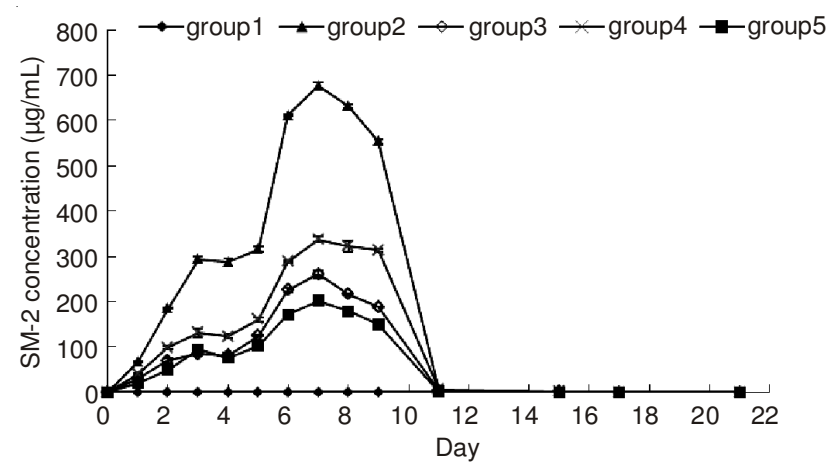

Fig. 2 concentration of SM-2 in swine slurry

Statistical analysis showed that the SM-2 concentration in group 2 was significantly higher than that of group 3 ( $p<$ $0.05)$ and that of group 4 was significantly higher than that of group $5(p<0.05)$ in SM-2 adding period. This indicated that the method of adding has direct impacts on the degradation of SM-2 during anaerobic digestion. This result maybe related to the inhibition of SM-2 and its metabolites on bacteria.

In addition, variance analysis showed that the concentration of SM-2 in group 2 was higher than that in group 4 $(p<0.05)$ and the same result as in group 3 and group $5(p$ $<0.05)$ in SM-2 adding period. It indicted that the degradation rate of SM-2 was related to the addition of SM-2 and addition of more SM-2 led to higher inhibition on microorganism and slower degradation of SM-2 in anaerobic digestion.

Methane production in anaerobic digestion: The methane production in anaerobic digestion systems was presented in Fig. 3. In SM-2 adding period, daily methane production in each group ranged from 120-170 $\mathrm{mL}$ and average methane production from group 1 to group 5 was $142.75 \pm 9.97,158.58$ $\pm 4.60,143.92 \pm 7.23,141.47 \pm 10.49,160.5 \pm 7.63 \mathrm{~mL}$ respectively. Variance analysis showed that there was no significant deference among all groups $(p>0.05)$. This result indicated that both the research method and the addition of SM-2 had no effect on the methane production in anaerobic digestion. To the present results, it seems to suggest that SM-2 has no inhibition on methanogenic archaea and thus methane production.

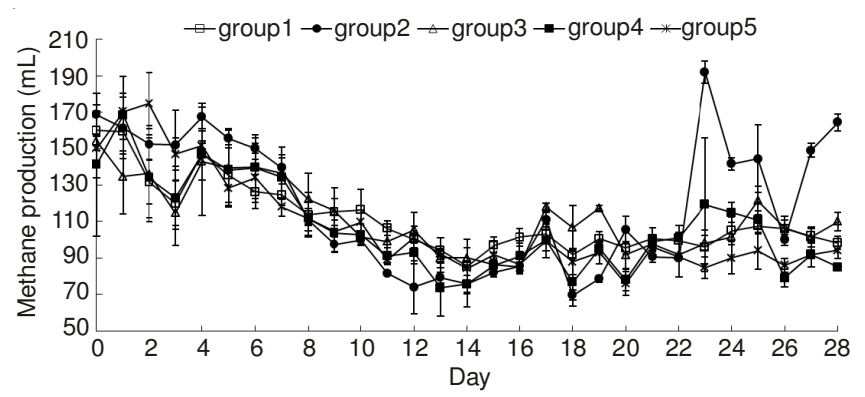

Fig. 3. Methane production in anaerobic digestion

Chemical properties of swine slurry: Effect of SM-2 on $\mathrm{pH}$ in anaerobic digestion slurry was shown in Fig. 4a. In SM-2 adding period, $\mathrm{pH}$ of swine slurry in both group 3 and group 5 were significantly higher than it in group $1(p<0.05)$ and there was no significant difference among the treatment groups $(p>0.05)$. But there was no significant difference in all groups during the entire experiment. It stated that adding SM-2 and blank manure could improve $\mathrm{pH}$ of swine slurry in adding SM-2 period.

Changes of COD concentration in anaerobic digestion were presented in Fig. 4b. There was no significant difference in SM-2 adding period, SM-2 withdrawing period and entire experimental period among all groups. It indicated that both methods and the addition of SM-2 had no effect on COD in anaerobic digestion.

Organic matter of biogas residue: The effect of SM-2 on organic matter of biogas residue was shown in Table-2. There was no significant difference among all groups $(p>$ $0.05)$. This result indicated that there was no significant effect of both the research method and the addition of SM-2 on organic matter of biogas residue.

Degradation of SM-2 in anaerobic digestion: Previous study showed that SM-2 could be degraded via photo-degradation and water-degradation. Microorganism can promote the degradation of SM- $2^{10}$. However, Zhang et al. ${ }^{11}$ reported that microbial degradation was an important approach to reduce sulfadiazine antibiotics in swine wastewater and high concentration of organic matter may promote microorganism degradation of antibiotics. This study was conducted under dark condition, thus photo-degradation had no effect on degradation of SM-2. The moisture content was $90 \%$ and organic matter 


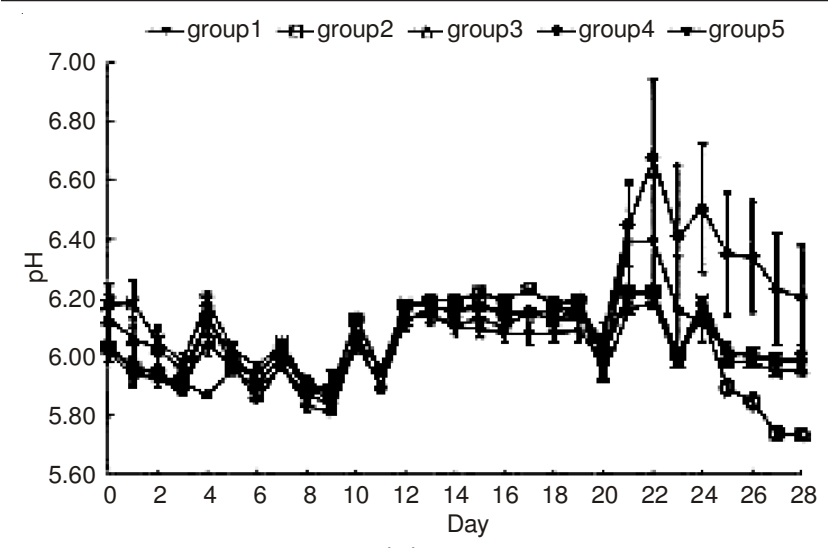

(a)

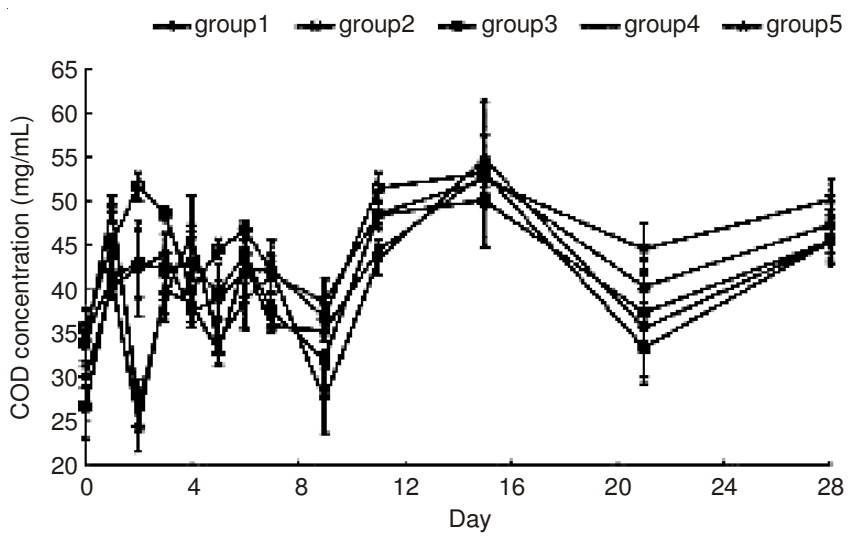

(b)

Fig. 4. Chemical properties during digesters in the anaerobic digestion

\begin{tabular}{|c|c|c|c|}
\hline \multicolumn{4}{|c|}{$\begin{array}{l}\text { ORGANIC MATTER CONCENTRATION OF BIOGAS } \\
\text { RESIDUE (MEAN } \pm \text { STD. ERROR, } \mathrm{g} / \mathrm{kg}, \mathrm{n}=3 \text { ) }\end{array}$} \\
\hline Day & 0 & 8 & 28 \\
\hline Group 1 & $672.61 \pm 14.98$ & $640.36 \pm 7.65$ & $649.19 \pm 26.04$ \\
\hline Group 2 & $653.47 \pm 15.80$ & $643.42 \pm 17.33$ & $645.63 \pm 2.88$ \\
\hline Group 3 & $644.09 \pm 18.63$ & $633.76 \pm 2.28$ & $672.99 \pm 2.57$ \\
\hline Group 4 & $644.98 \pm 7.32$ & $646.46 \pm 3.42$ & $653.89 \pm 8.87$ \\
\hline Group 5 & $679.80 \pm 16.01$ & $665.70 \pm 22.22$ & $630.85 \pm 13.83$ \\
\hline
\end{tabular}

concentration was high in anaerobic digestion, thus we believed water-degradation and microbial degradation were the major approaches to the degradation of SM-2.

The concentration of SM-2 in the treatment using manure from medicated swine was significant higher than that in the treatment adding with SM-2 and antibiotic-free manure. It is mainly because that medicated swine manure contains both SM-2 and its metabolites and that might reduce the degradation of microorganism on SM-2 more efficiently than SM-2 itself. Besides that, microorganism might lead to the transformation from the metabolites of SM-2 to the original form of SM-2 in anaerobic digestion ${ }^{12}$.

The concentration of SM-2 increased steadily in SM-2 adding period in anaerobic digestion. The experimental temperature, moisture content and $\mathrm{pH}$ were kept the same in all groups, so we can believe that they might not affect the degradation of SM-2. Since SM-2 is a broad-spectrum antibiotic, the population of microorganism in the anaerobic system may be inhibited by the SM-2, so that SM-2 was accumulated in anaerobic digestion with the consecutive addition of SM-2. As swine slurry containing SM-2 was displaced with blank swine manure consecutively in SM-2 withdrawing period, the concentration of SM-2 gradually decreased and microbial activity restored to promote water-degradation and microbialdegradation, so that the concentration of SM-2 reduced gradually until it was undetectable on the 11th day. Shi et al. ${ }^{9}$ observed that the concentration of orosulfan rapidly reduced to $50 \%$ within the first $12 \mathrm{~h}$, then slow down until it was not be detected on the 20th day under two adding concentrations ( 25 and $50 \mathrm{mg} / \mathrm{L}$ ) in anaerobic digester. The different result was because in this study the concentration of SM-2 in the anaerobic digestion was lower (12.82 and $24.25 \mathrm{mg} / \mathrm{L}$ ) than those in the study of Shi et al. ${ }^{9}$. In addition, this study was conducted using a semi-batch model and as the result SM-2 in anaerobic digestion system was diluted by blank manure in SM-2 withdrawing period.

Effect of SM-2 on anaerobic digestion: Massé et al. ${ }^{4}$ added $110 \mathrm{mg} /(\mathrm{kg} \mathrm{d} \mathrm{m}) \mathrm{SM}-2$ into pig diet and collected pig manure and urine without detecting concentration of SM-2 and they found that there was no significant effect of SM-2 on methane production, $\mathrm{pH}$ and COD under single addition. However, Loftin et al. ${ }^{13}$ reported that the direct addition of 10 $\mathrm{mg} / \mathrm{L} \mathrm{SM}-2$ in pig manure could significantly inhibit methane production but not so as the COD. In this study, we adopted the semi-batch model that was similar to the farm conditions which SM-2 entered anaerobic digester consecutively in medicated period. The result showed that both the experimental concentration and the research method had no effect on the indexes of anaerobic digestion such as methane and COD which agreed with several previous study ${ }^{4}$.

This study indicated that $\mathrm{pH}$ of swine slurry was improved in the treatment added SM-2 and blank manure with feeding SM-2. It was possible because that the acidogenic bacteria had been resistant to SM-2 in medicated swine manure, but adding of SM-2 and blank manure into anearobic degestion could probably inhitied acidogenic bacteria and increase the $\mathrm{pH}$ of slurry. Then with the adaptation of acidogenic bacteria to SM-2, the inhition reduced and $\mathrm{pH}$ gradually recoverded.

Sankvist et al. ${ }^{7}$ concluded that using manure of medicated swine with veterinary antibiotic could inhibit methane produc-tion but not probable as direct adding when manure and veterinary antibiotic were added directly. However, this study showed that the two research methods had no significant effect on the methane production in anaerobic digestion. The primary reason could be because this study was adopting a semi-batch system that was different from previous study methods which were disposable adding veterinary antibiotic into static anaerobic digestion. Furthermore, Loftin et al. ${ }^{13}$ believed that the wide use of veterinary antibiotics in swine farm could induce the microbial resistance and maybe reduce the inhibition on methane production, so the effect of veterinary antibiotics on anaerobic digestion was no longer significant as in previous studies.

\section{Conclusion}

Inconsistencies exist among results of different research methods on the effects of SM-2 in anaerobic fermentation of 
swine manure slurry. However, we conclude that using manure collect from animals administered with SM-2 is more appropriate to assess the effect of SM-2 on anaerobic digestion.

\section{REFERENCES}

1. M. Mellon, C. Benbrook and K.L. Benbrook Estimates of Antibiotics Abuse in livestock: Union of Concerned, Scientists Publications Washington DC, pp. 7-9 (2001).

2. L. Zhao, Y.H. Dong and H. Wang, Sci. Total Environ., 408, 1069 (2010).

3. C.B. Fedler and D.L. Day, Trans. ASAE, 28, 523 (1985).

4. D.I. Massé, D.L. Lu, L. Masse and R.L. Droste, Bioresour. Technol., 75, 202 (2000)

5. O.A. Arikan, L.J. Sikora, W. Mulbry, S.U. Khan, C. Rice and G.D. Foster, Process Biochem., 41, 1637 (2006).
6. J.J. Stone, S.A. Clay, Z. Zhu, K.L. Wong, L.R. Porath and G.M. Spellman, Water Res., 43, 4740 (2009).

7. A. Sankvist, M. Hagelberg and B. Mathisen, Bioenergy, 84, 422 (1984).

8. Commission of Chinese Veterinary Pharmacopoeia, Veterinary Pharmacopoeia of the People Republic of China, China Agriculture Press (2005).

9. J.C. Shi, X.D. Liao, Y.B. Wu and J.B. Liang, An. Feed Sci. Technol., 166-167, 457 (2011)

10. J.-P. Niu, Z.-H. Wu and Q.-Z. Shi, J. Anhui Agric. Sci., 37, 1767 (2009).

11. C.L. Zhang, Y. Wang and F. Wang, Ecol. Environ., 16, 1679 (2007).

12. L.L. Duan, Fate of Sulfamethazine and Its Metabolite in Sandy Loamy Soil, China Agriculture University (2005).

13. K.A. Loftin, C. Henny, C.D. Adams, R. Surampali and M.R. Mormile, Environ. Toxicol. Chem., 24, 782 (2005). 